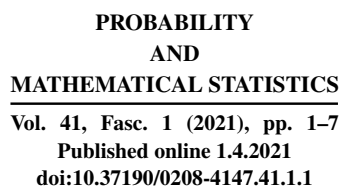

\title{
ON MIXTURES OF GAMMA DISTRIBUTIONS, DISTRIBUTIONS WITH HYPERBOLICALLY MONOTONE DENSITIES AND GENERALIZED GAMMA CONVOLUTIONS (GGC)
}

BY

TORD S JÖDIN (UMEÅ)

\begin{abstract}
Let $Y$ be a standard Gamma( $(k)$ distributed random variable (rv), $k>0$, and let $X$ be an independent positive rv. If $X$ has a hyperbolically monotone density of order $k\left(\mathrm{HM}_{k}\right)$, then $Y \cdot X$ and $Y / X$ are generalized gamma convolutions (GGC). This extends work by Roynette et al. and Behme and Bondesson. The same conclusion holds with $Y$ replaced by a finite sum of independent gamma variables with sum of shape parameters at most $k$. Both results are applied to subclasses of GGC.
\end{abstract}

2020 Mathematics Subject Classification: Primary 60E10; Secondary $62 \mathrm{E} 15$.

Key words and phrases: gamma distribution, hyperbolically monotone function, Laplace transform, generalized gamma convolution (GGC).

\section{INTRODUCTION}

We consider classes of distributions on the positive real axis. Among these are gamma distributions, distributions with hyperbolically monotone densities $\left(\mathrm{HM}_{k}\right)$, distributions with hyperbolically completely monotone densities (HCM) and generalized gamma convolutions (GGC). Generalized gamma convolutions were introduced by O. Thorin [8], [9] in his study of infinite divisibility of the log-normal distribution. The class GGC consists of limit distributions of convolutions of independent gamma distributions and is closed with respect to (wrt) weak limits and sums and products of independent rvs. A comprehensive study of GGC and its relation to HCM can be found in [2] and [3]. For more on Bernstein functions and infinite divisibility, see [6] and [7].

Recently, Behme and Bondesson [1] generalized a result of Roynette et al. [5] on products $Y \cdot X$ and quotients $Y / X$ of a gamma distributed variable $Y$ and an independent rv $X$ with hyperbolically monotone density. More exactly, they proved that if $0<r \leqslant k, k$ is a positive integer, $Y \sim \operatorname{Gamma}(r)$ and $X \sim \mathrm{HM}_{k}$, then $Y \cdot X \sim$ GGC and $Y / X \sim$ GGC [1, Theorem 1 and Corollary 1], while 
Roynette et al. [5] proved the case $k=1$. It was conjectured in [1] that this result remains true for all $k>0$. We confirm this conjecture and prove that if $Y \sim \operatorname{Gamma}(k)$ and $X \sim \mathrm{HM}_{k}$ are independent rvs, then $Y \cdot X \sim \mathrm{GGC}$ and $Y / X \sim$ GGC, for all $k>0$ (Theorem 3.1). In addition, we prove the same result when $Y$ is replaced by a finite sum of independent gamma variables with the sum of the shape parameters at most $k$ (Theorem 4.1). The proofs are based on Bondesson's characterization of GGC and explicit integral expressions for the relevant functions. The results are applied to subclasses of GGC (Theorems 5.1 and 5.2).

The plan of this paper is as follows. Section 2 begins with the standard notation and definitions used in this field and reviews a part of the set up in [1, Section 3]. We describe our approach in Section 3 and state and prove our main result (Theorem 3.1). The last two sections contain applications and comments.

\section{PRELIMINARIES}

This section gives the background to our results and defines the concepts that are needed to state and prove our theorems. A more complete presentation of this field and a survey of many of its results can be found in [2] and [1]. A function $f(x)$ is called hyperbolically monotone $\left(\mathrm{HM}_{1}\right)$ if, for every fixed $u>0, h(w)=f(u v)$. $f(u / v)$ is nonincreasing as a function of $w=v+v^{-1}$. If $k$ is a positive integer, $f$ is called hyperbolically monotone of order $k\left(\mathrm{HM}_{k}\right)$ if $(-1)^{j} \cdot D^{j} h(w) \geqslant 0$ for $j=1, \ldots, k-1$, and $(-1)^{k-1} \cdot D^{(k-1)} h(w)$ decreases. Finally, we say that $f$ is hyperbolically completely monotone if this holds for all positive integers $k$, or equivalently $h(w)$ is completely monotone $(\mathbf{C M})$ with respect to $w$. See [2, Ch. 5] and [1] for more on these classes. It is proved in [1, Section 2] that a density function $f(x)$ is $\mathrm{HM}_{k}$ if and only if, for every $u>0$,

$$
h(w)=f(u v) \cdot f(u / v)=\int_{(w, \infty)}(\lambda-w)^{k-1} H_{u}(d \lambda)
$$

for $w=v+v^{-1}$ and some nonnegative measure $H_{u}(d \lambda)$ depending on $u$. Following [2] we take (2.1) as our definition of $\mathrm{HM}_{k}, k>0$.

Definition 2.1. A rv $X \sim \mathrm{HM}_{k}$ if $X$ has density $f(x)$ satisfying (2.1).

We let $\operatorname{Gamma}(k, r)$ denote the standard class of gamma distributions with density $f(x)=r^{k} \cdot \Gamma(k)^{-1} \cdot x^{k-1} \cdot e^{-r x}, x>0$, Gamma $(k)=\operatorname{Gamma}(k, 1)$. A generalized gamma convolution (GGC) is a probability distribution $F$ on $[0, \infty)$ with Laplace transform

$$
\phi(s)=\int e^{-s x} F(d x)=\exp \left(-a s+\int \log \left(\frac{t}{t+s}\right) U(d t)\right), \quad s \geqslant 0,
$$

where $a \geqslant 0$ (called the left extremity) and $U(d t)$ is a nonnegative measure on $(0, \infty)$, with finite mass on all compact subintervals, such that $\int_{0}^{1}|\log t| U(d t)$ $<\infty$ and $\int_{1}^{\infty} t^{-1} U(d t)<\infty[2$, Ch. 3]. 
In the following we prepare for the proof of Theorem 3.1 and follow the presentation in [1, Section 3]. Let $X \sim \operatorname{HM}_{k}$ with density $f(x)$ and $Y \sim \operatorname{Gamma}(k, r)$ be independent rvs. Then the Laplace transform $\phi(s)$ of the quotient $Y / X$ is given by

$$
\phi(s)=E\left[e^{-s Y / X}\right]=\int_{0}^{\infty} E\left[e^{-s Y / x}\right] \cdot f(x) d x=\int_{0}^{\infty}\left(\frac{r x}{r x+s}\right)^{k} \cdot f(x) d x
$$

and for any $s, t>0$ we write $\phi(s t) \phi(s / t)$ as a product of two such integrals and get

$$
\begin{aligned}
\phi(s t) \phi & (s / t)=\int_{0}^{\infty} \int_{0}^{\infty}\left(\frac{r^{2} x y}{(r x+s t)(r y+s / t)}\right)^{k} \cdot f(x) f(y) d x d y \\
& =\int_{0}^{\infty} \int_{0}^{\infty} \frac{2 u}{v}\left(\frac{r^{2} u^{2}}{(r u v+s t)(r u / v+s / t)}\right)^{k} \cdot f(u v) f(u / v) d u d v \\
& =\int_{0}^{\infty} \int_{0}^{\infty} \frac{2 u}{v}\left(\frac{r^{2} u^{2}}{(r u v+s t)(r u / v+s / t)}\right)^{k} \cdot \int_{(w, \infty)}(\lambda-w)^{k-1} H_{u}(d \lambda) d u d v
\end{aligned}
$$

after the standard hyperbolic change of variables $x=u v, y=u / v$, with Jacobian $-2 u / v$, and inserting the definition (2.1) of $f$ being $\mathrm{HM}_{k}$. Next we fix an arbitrary $u>0$ and $\lambda=b+b^{-1} \geqslant 2$ for some $b \geqslant 1$. Changing the order of integration, this gives integration with respect to $v$ over the interval $\left[b^{-1}, b\right]$ in the innermost integral

$$
\int_{1 / b}^{b}\left(\frac{r^{2} u^{2}}{(r u v+s t)(r u / v+s / t)}\right)^{k} \cdot\left(b+\frac{1}{b}-v-\frac{1}{v}\right)^{k-1} \frac{d v}{v} .
$$

We let $b>1$ and note that $b+b^{-1}-v-v^{-1}=(b-v)\left(v-b^{-1}\right) / v$. After some algebra and putting $r u / s=a$ we find that a sufficient condition for $Y / X \sim$ GGC is that the integral

$$
J_{k}=\int_{1 / b}^{b}\left(\frac{(b-v)\left(v-b^{-1}\right)}{(v+t a)(v+t / a)}\right)^{k} \cdot \frac{t^{k}}{(b-v)\left(v-b^{-1}\right)} d v
$$

is CM with respect to $T=t+1 / t$, for all $a>1$ and $b>1$. From now on we assume that $a$ and $b$ are fixed but arbitrary such numbers. The substitution $v \mapsto v^{-1}$ confirms that the map $t \mapsto t^{-1}$ leaves $J_{k}$ invariant and that $J_{k}$ is a function of $T$.

The integrand in (2.2) is a rational function when $k$ is a positive integer and $J_{k}$ can, at least in principle, be calculated explicitly. A straightforward calculation gives $J_{1}=\left(a-a^{-1}\right)^{-1} \cdot \log ((T+A) /(T+B))$, where $A=a b+(a b)^{-1}$ and $B=a b^{-1}+a^{-1} b$, and it is easily seen that $J_{1}$ is $\mathrm{CM}$ with respect to $T$, since 
$A>B$. In the general integer case, $J_{k}$ is expressed in terms of certain polynomials $P_{k}$ and $Q_{k}$ and the logarithm above [1, Theorem 1]. Our proof for $k>0$ is based on a transformation of the integral $J_{k}$ to a new form that is more suitable for our purposes and follows quite different lines.

\section{MAIN RESULT}

In this section we state and prove our first result that products and quotients of independent rvs $Y \sim \operatorname{Gamma}(k)$ and $X \sim \mathrm{HM}_{k}, k>0$, have GGC distributions. This result generalizes, respectively, the cases $k=1$ in [5] and $k$ a positive integer in [1], to arbitrary $k>0$.

THEOREM 3.1. Let $0<k \leqslant l$ and let $Y \sim \operatorname{Gamma}(k)$ and $X \sim \mathrm{HM}_{l}$ be independent rvs. Then $Y \cdot X \sim \mathrm{GGC}$ and $Y / X \sim \mathrm{GGC}$.

It is sufficient to prove Theorem 3.1 for $k=l$, since $\mathrm{HM}_{l} \subseteq \mathrm{HM}_{k}$ for $0<k \leqslant l$ [1, Section 5].

Proof. Since $X \sim \mathrm{HM}_{k}$ if and only if $1 / X \sim \mathrm{HM}_{k}$ it is enough to prove that $Y / X \sim$ GGC. We evaluate $J_{k}$ by a series of substitutions and start with

$$
v=\left(b x+b^{-1}\right)(1+x)^{-1}, \quad d v / d x=\left(b-b^{-1}\right) \cdot(1+x)^{-2}
$$

and $(b-v)\left(v-b^{-1}\right)=\left(b-b^{-1}\right)^{2} x \cdot(1+x)^{-2}$. Then $J_{k}=\int_{0}^{\infty} E(x) \frac{d x}{x}$, where

$$
E(x)=\left(\frac{\left(b-b^{-1}\right)^{2}}{\left(b^{2} x+\frac{1}{b^{2} x}\right) \cdot \frac{1}{t}+2 T+\left(x+\frac{t}{x}\right) \cdot t+\left(b x+\frac{1}{b x}+\beta\right) \cdot \alpha}\right)^{k} \cdot\left(b-b^{-1}\right)^{-1},
$$

where $\alpha=a+a^{-1}$ and $\beta=b+b^{-1}$. The denominator $D$ inside the parentheses in $E(x)$ can be rewritten as

$$
D=x \cdot\left(\frac{b^{2}}{t}+t+\alpha b\right)+\frac{1}{x} \cdot\left(\frac{1}{b^{2} t}+t+\frac{\alpha}{b}\right)+2 T+\alpha \beta,
$$

and a standard substitution, making the second term in $D$ equal to $1 / \rho$, transforms $D$ into

$$
D_{1}=\rho^{-1}+\rho \cdot(T+A)(T+B)+2 T+\alpha \beta,
$$

where $A=a b+(a b)^{-1}, B=a b^{-1}+a^{-1} b$. Now let $\delta=\rho \cdot \sqrt{(T+A)(T+B)}$ and we get

$$
J_{k}=\int_{0}^{\infty} \frac{\left(b-b^{-1}\right)^{2 k}}{\left(\left(\delta+\frac{1}{\delta}\right) \sqrt{(T+A)(T+B)}+2 T+\alpha \beta\right)^{k}} \cdot\left(b-b^{-1}\right)^{-1} \frac{d \delta}{\delta},
$$


which is our final expression for $J_{k}$. This is the transformation of $J_{k}$ mentioned at the end of Section 2. The expression inside the parentheses in the denominator in (3.1) is now easily seen to have a CM derivative, since by direct calculation

$$
-\frac{d^{2}}{d T^{2}} \sqrt{(T+A)(T+B)}=\frac{1}{4} \cdot \frac{(A-B)^{2}}{(T+A)^{3 / 2} \cdot(T+B)^{3(2}}
$$

is CM with respect to $T$. Composing that expression with the negative power function proves that $J_{k}$ is CM with respect to $T$ [4, Criterion 2, p. 441]. The proof of Theorem 3.1 is complete.

\section{SUMS OF INDEPENDENT GAMMA VARIABLES}

In this section we replace the gamma variable $Y$ in Theorem 3.1 by a finite sum of independent gamma variables with sum of shape parameters at most $k$, as was suggested in [1, Section 5]. We prove that the conclusions of Theorem 3.1 remain true (Theorem 4.1) and apply the result to subclasses of GGC in the next section.

THEOREM 4.1. Let $Y=Y_{1}+\cdots+Y_{n}$ be a finite sum of independent gamma variables, $Y_{i} \sim \operatorname{Gamma}\left(k_{i}, r_{i}\right), 1 \leqslant i \leqslant n$, such that $k_{1}+\cdots+k_{n} \leqslant k$, and let $X \sim \mathrm{HM}_{k}$ be an independent $r v$. Then $Y \cdot X \sim \mathrm{GGC}$ and $Y / X \sim \mathrm{GGC}$.

Proof. As noted above, it is no loss of generality to assume $k_{1}+\cdots+k_{n}=k$. Let $Y$ and $X$ be as in the theorem. Then as above

$$
\phi(s)=E\left[e^{-s Y / X}\right]=\int_{0}^{\infty} E\left[e^{-s Y / x}\right] \cdot f(x) d x=\int_{0}^{\infty} \prod_{i=1}^{n}\left(\frac{r_{i} x}{r_{i} x+s}\right)^{k_{i}} \cdot f(x) d x
$$

and

$\phi(s t)\left(\phi(s / t)=\int_{0}^{\infty} \int_{0}^{\infty} \frac{2 u}{v} \prod_{i=1}^{n}\left(\frac{r_{i}^{2} u^{2}}{\left(r_{i} u v+s t\right)\left(r_{i} u / v+s / t\right)}\right)^{k_{i}} \cdot f(u v) f(u / v) d u d v\right.$.

We now proceed as in the proof of Theorem 3.1 and find that it is sufficient to prove that the following analogue of (2.2),

$$
J_{k}=\int_{1 / b}^{b} \prod_{i=1}^{n}\left(\frac{(b-v)(v-1 / b)}{\left(v+t a_{i}\right)\left(v+t / a_{i}\right)}\right)^{k_{i}} \cdot \frac{t^{k}}{(b-v)(v-1 / b)} d v
$$

is $\mathrm{CM}$ with respect to $T=t+t^{-1}$, where we define $a_{i}=r_{i} u / s, 1 \leqslant i \leqslant n$. Then the same substitution $v=\left(b x+b^{-1}\right)(1+x)^{-1}$ as above gives

$$
J_{k}=\int_{0}^{\infty} \prod_{i=1}^{n} \frac{\left(b-b^{-1}\right)^{2 k_{i}}}{\left(x \cdot\left(\frac{b^{2}}{t}+t+\alpha_{i} b\right)+\frac{1}{x} \cdot\left(\frac{1}{b^{2} t}+t+\frac{\alpha_{i}}{b}\right)+2 T+\alpha_{i} \beta\right)^{k_{i}}} \cdot \frac{1}{b-b^{-1}} \frac{d x}{x} .
$$


An immediate change of the $x$-variable does not work here, since each factor would require its special substitution. We turn to the Laplace transform instead. Denote by $S_{i}(x)$ the expression inside the parentheses of the $i$ th factor in the denominator of the integrand. Then the integrand is $\mathrm{CM}$ with respect to $S_{i}(x), 1 \leqslant i \leqslant n$, and by Bernstein's theorem there is a nonnegative measure $\nu(d \lambda)$ such that $J_{k}=$ $\int_{0}^{\infty} \frac{d x}{x} \int_{0}^{\infty} \nu(d \lambda) e^{-E(x)}$, where $E(x)=\sum_{i=1}^{n} \lambda_{i} S_{i}(x)$. Next we change the order of integration in $J_{k}$, denote the inner integral by $I_{k}$ and set out to prove that $I_{k}$ is $\mathrm{CM}$ with respect to $T$, for all choices of $\lambda_{i}, 1 \leqslant i \leqslant n$. Assume that $\sum_{i=1}^{n} \lambda_{i}=p$, define $\alpha$ by $\sum_{i=1}^{n} \lambda_{i} \alpha_{i}=p \cdot \alpha$ and choose $a>1$ such that $\alpha=a+a^{-1}$. Then

$$
\frac{1}{p} \cdot E(x)=x \cdot\left(\frac{b^{2}}{t}+t+\alpha b\right)+\frac{1}{x} \cdot\left(\frac{1}{b^{2} t}+t+\frac{\alpha}{b}\right)+2 T+\alpha \beta
$$

and performing the same substitutions as in the proof of Theorem 3.1 gives

$$
I_{k}=\int_{0}^{\infty} e^{-p \cdot\left(\left(\delta+\frac{1}{\delta}\right) \sqrt{(T+A)(T+B)}+2 T+\alpha \beta\right)} \frac{d \delta}{\delta},
$$

with $\alpha, p$ and $a$ defined above and $A=a b+(a b)^{-1}, B=a b^{-1}+a^{-1} b$. This shows that $J_{k}$ is CM with respect to $T$, since the integrand is the composition of a negative exponential function and a nonnegative function with a $\mathrm{CM}$ derivative [4, Criterion 2, p. 441]. This completes the proof of Theorem 4.1.

\section{SOME CONSEQUENCES}

Let $\mathcal{A}$ and $\mathcal{B}$ be classes of distributions and denote by $\mathcal{A} \times \mathcal{B}$ the class of products $Y \cdot X$ of independent rvs $Y \sim \mathcal{A}$ and $X \sim \mathcal{B}$, and similarly for quotients. Then $\operatorname{Gamma}(k) \times \mathrm{HM}_{k} \subset \mathrm{GGC}$ and $\operatorname{Gamma}(k) / \mathrm{HM}_{k} \subset \mathrm{GGC}$, for $k>0$, by Theorem 3.1. If $Z \sim \mathrm{GGC}$ is independent of $Y$ and $X$, then $(Z \cdot Y) \cdot X=Z \cdot(Y \cdot X)$ $\sim$ GGC and $(Z \cdot Y) / X=Z \cdot(Y / X) \sim \mathrm{GGC}$, since GGC is closed with respect to independent products [3, Theorem 1], and we have the following result.

Theorem 5.1. For any $k>0,(\operatorname{GGC} \times \operatorname{Gamma}(k)) \times \mathrm{HM}_{k} \subset \mathrm{GGC}$ and $(\operatorname{GGC} \times \operatorname{Gamma}(k)) / \mathrm{HM}_{k} \subset \mathrm{GGC}$.

If $\mathcal{H}_{k}$ is the largest class of probability distributions such that $\operatorname{Gamma}(k) \times \mathcal{H}_{k}$ $\subset \mathrm{GGC}$, then $\mathrm{HM}_{k} \times \mathrm{GGC} \subset \mathcal{H}_{k}$. Similarly, if $\mathcal{G}_{k}$ is the largest class of probability distributions such that $\mathcal{G}_{k} \times \mathrm{HM}_{k} \subset \mathrm{GGC}$, then $\mathrm{GGC} \times \operatorname{Gamma}(k) \subset \mathcal{G}_{k}$. We can use Theorem 4.1 to improve on this. Let $\mathrm{GGC}(k)$ be the class of rvs $X \sim \mathrm{GGC}$ with left extremity zero and total $U$-measure at most $k$.

THEOREM 5.2. For any $k>0, \operatorname{GGC}(k) \subset \mathcal{G}_{k}$.

Proof. Every $X \sim \mathrm{GGC}(k)$ is the weak limit of finite sums of independent gamma variables with $U$-measure at most $k$ and the conclusion follows from Theorem 4.1. 


\section{FINAL COMMENTS}

The extension of the integer case of [1, Theorem 1] to hold for all $k \geqslant 1$, and even for all $k>0$, was suggested to me by Professor Bondesson. Our proof shows that, in contrast to what is believed in [1], the function $J_{k}$ in (3.1) can be explicitly calculated also in the noninteger case. The truth of Theorem 4.1 and the possibility that $\operatorname{GGC}(k)$ is contained in $\mathcal{G}_{k}$ are mentioned in [1, Section 5].

Acknowledgments. The author thanks Professor Lennart Bondesson for introducing him to the problems studied here and for valuable comments and discussions.

\section{REFERENCES}

[1] A. Behme and L. Bondesson, A class of scale mixtures of gamma $(k)$-distributions that are generalized gamma convolutions, Bernoulli 23 (2017), 773-787.

[2] L. Bondesson, Generalized Gamma Convolutions and Related Classes of Distributions and Densities, Lecture Notes in Statist. 76, Springer, New York, 1992.

[3] L. Bondesson, A class of probability distributions that is closed with respect to addition as well as multiplication of independent random variables, J. Theoret. Probab. 28 (2015), 1063-1081.

[4] W. Feller, An Introduction to Probability Theory and its Applications, Vol. II, Wiley, New York, 1966.

[5] B. Roynette, P. Vallois, and M. Yor, A family of generalized gamma convoluted variables, Probab. Math. Statist. 29 (2009), 181-204.

[6] R. L. Schilling, R. Song, and Z. Vondraček, Bernstein Functions, de Gruyter Stud. Math. 37, de Gruyter, Berlin, 2010.

[7] F. W. Steutel and K. van Harn, Infinite Divisibility of Probability Distributions on the Real Line, Dekker, New York, 2004.

[8] O. Thorin, On the infinite divisibility of the lognormal distribution, Scand. Actuarial J. 1977, 121-148.

[9] O. Thorin, An extension of the notion of a generalized $\Gamma$-convolution, Scand. Actuarial J. 1978, 141-149.

Tord Sjödin

Department of Mathematics and

Mathematical Statistics

Umeå University

90187 Umeå, Sweden

E-mail: tord.sjodin@umu.se

Received 14.12.2018;

revised version 17.12.2019 
